COMMENT. The authors list three novel findings: 1) the quantifiable delay in early development of infants with sagittal synostosis (SS) demonstrated in a controlled, prospective, longitudinal study; 2) a preoperative delay in gross locomotor development; and 3) a significant postoperative resolution of locomotor deficits. Previous studies have reported developmental delay only in older patients and without normal controls and prospective follow-up; mental rather than motor development has been considered; and delayed gross locomotor development with improvement after surgery has not previously been demonstrated in young infants. One study is cited that supports the present findings but in young children: $35.5 \%$ of 50 children with SS had cerebral palsy, psychomotor retardation, and/or neurological signs, and many of the deficits resolved after surgery, only $14.5 \%$ having persistent abnormal signs (Kaiser G. Childs Nerv Syst 1988;4:223-230). Raised intracranial pressure was considered an unlikely factor in the mechanism of improved locomotor function following surgery in the Leeds series.

\title{
BEHAVIORAL AND SLEEP DISORDERS IN NEUROFIBROMATOSIS
}

The behavior and sleep patterns of 64 children (mean age 10 years 7 months) with neurofibromatosis type 1 (NF1) were determined by mail and telephone questionnaire in a study at Park Hospital, University of Oxford, UK. Compared to the general population, increased numbers of children with NF1 had scores in the borderline and abnormal range for peer problems $(p<0.001)$, hyperactivity $(p<0.001)$, emotional symptoms $(p<0.001)$, and conduct disorders $(\mathrm{p}<0.05)$. Parasomnias (sleepwalking and sleep terrors) were more frequent in the NF1 patients $(p<0.05)$, and those with frequent sleep disturbance had a higher incidence of conduct, hyperactivity, emotional, and total behavioral disorders $(\mathrm{p}<0.05$ or 0.01). (Johnson H, Wiggs L, Stores G, Huson SM. Psychological disturbance and sleep disorders in children with neurofibromatosis type 1. Dev Med Child Neurol April 2005;47:237-242). (Respond: Dr Hilary Johnson, Park Hospital for Children, Old Road, Headington, Oxford OX3 7LQ, UK).

COMMENT. NF1 is associated with an increased prevalence of behavioral and sleep problems, including attention deficit hyperactivity disorder.

Cognitive impairments and specific learning disabilities occur in $30-65 \%$ of patients with NF1, and the association is reviewed by Ward BA and Gutmann DH (Pediatr Neurol April 2005;32:221-228). These authors cite several studies showing a correlation between unidentified bright objects (T2 hyperintensities) in the brain MRI and cognitive dysfunction, but the observation is controversial and not universal. The role of neurofibromin, the NF1 gene product, in learning is discussed in relation to laboratory and genetic studies in $\mathrm{Nfl}+1$ mice. The increased RAS proto-oncogene activity in these mice is responsible for a spatial learning impairment that is related to $g$-aminobutyric acid-mediated inhibition. Active RAS, as a result of reduced neurofibromin, leads to tumor formation in NF1. Experimental therapies in trial phase, such as farnesyl transferase inhibitors that decrease RAS levels, are intended to decrease tumor size and these may lead to a reversal of the learning disability. For further articles on NF1 and learning disabilities, see Progress in Pediatric Neurology 111, PNB Publishers, 1997;pp291-294, 441. 